\title{
Effectiveness of topical budesonide in preventing esophageal strictures after endoscopic resection of esophageal cancer
}

\section{다 (우우}

\author{
Authors \\ Steffi Elisabeth Maria van de Ven, Manon J.B.L. Snijders, Marco J. Bruno, Arjun Dave Koch
}

Institution

Department of Gastroenterology and Hepatology, Erasmus

MC, University Medical Center, Rotterdam, The Netherlands

submitted 2.6.2020

accepted after revision 3.8 .2020

Bibliography

Endoscopy International Open 2020; 08: E1795-E1803

DOI 10.1055/a-1266-3423

ISSN 2364-3722

(C) 2020. The Author(s).

This is an open access article published by Thieme under the terms of the Creative Commons Attribution-NonDerivative-NonCommercial License, permitting copying and reproduction so long as the original work is given appropriate credit. Contents may not be used for commecial purposes, or adapted, remixed, transformed or built upon. (https://creativecommons.org/licenses/by-nc-nd/4.0/)

\section{Corresponding author}

Arjun D. Koch, Postbox 2040, 3000 CA, Rotterdam, the Netherlands

Fax: + 31107040566

a.d.koch@erasmusmc.nl

Supplementary material is available under https://doi.org/10.1055/a-1266-3423

\section{ABSTRACT}

Background and study aims A disadvantage of endoscopic resection (ER) of early esophageal cancer (EC) is the high stricture rate after resection. A risk factor for stricture development is a mucosal defect after ER of $\geq 75 \%$ of the esophageal circumference. Stricture rates up to $94 \%$ have been reported in these patients. The aim of this study was to investigate the effectiveness of oral treatment with topical budesonide for stricture prevention after ER of early EC. Patients and methods We performed a retrospective analysis of a prospective cohort study of patients who received topical budesonide after ER of EC between March 2015 and April 2020. The primary endpoint was the esophageal stricture rate after ER. Stricture rates of our cohort were compared with stricture rates of control groups in the literature.

Results In total, 42 patients were treated with ER and topical budesonide. A total of 18 of 42 patients (44.9\%) developed a stricture. The pooled stricture rate of control groups in the literature was $75.3 \%$ (95\% Cl 68.8\%-81.9\%). Control groups consisted of patients with esophageal squamous cell carcinoma with a mucosal defect after ER of $\geq 75 \%$ of the esophageal circumference. Comparable patients of our cohort had a lower stricture rate $(47.8 \%$ vs. $75.3 \%, P=$ 0.007).

Conclusions Topical budesonide therapy after ER for EC seems to be a safe and effective method in preventing strictures. The stricture rate after budesonide treatment is lower compared to the stricture rate of patients who did not receive a preventive treatment after ER reported in the literature.

\section{Introduction}

Over the past few decades, the incidence of esophageal cancer (EC) has increased [1]. The most common histological type of EC is esophageal squamous cell carcinoma (ESCC) [2]. The overall survival of patients with EC has improved due to better treatment options such as neoadjuvant chemoradiotherapy, and the treatment shift from esophagectomy to endoscopic resection (ER) for early EC [3-5].

Compared to surgery, ER has lower morbidity and mortality for early EC while maintaining equal curative outcomes [5].
Endoscopic mucosal resection (EMR) and endoscopic submucosal dissection (ESD) are established treatment options when EC is limited to the superficial layers [6]. ESD is recommended over EMR for a selected number of adenocarcinomas larger than 15 $\mathrm{mm}$ with suspected submucosal invasion, and for all superficial ESCCs except if submucosal invasion is suspected [7]. Moreover, ESD provides en bloc resection and it reduces the number of recurrences compared to EMR $[8,9]$.

A major disadvantage of ER of large esophageal tumors, however, is the high stricture rate after resection $[10,11]$. Stricture formation after ER of early EC with a mucosal defect $\geq 75 \%$ 
of the esophageal circumference is reported up to $94.1 \%$ in the literature [10]. Esophageal strictures develop as a result of inflammation in the wound healing process of the mucosal defect following ER [12]. Previous studies have shown that a mucosal defect of $\geq 75 \%$ of the esophageal circumference was associated with esophageal stricture rates of $70 \%$ to $94 \%$ and it is reported as a significant risk factor for stricture development $[10,13-15]$. Patients with strictures might suffer from dysphagia with the need for endoscopic dilations, and patient quality of life might substantially decrease [16]. In addition, endoscopic dilations are associated with an increased perforation risk [17].

In an effort to reduce the stricture rate after ER of esophageal tumors, preventive strategies have been investigated [18]. For example, treatment with triamcinolone injections, the use of polyglycolic acid sheet (PGA) with fibrin glue, preventive endoscopic balloon dilation (EBD), and treatment with oral prednisolone [16,19-24]. Although these studies have shown promising results in preventing strictures after ESD, limited patients were included and the optimal dose and duration of steroids has not yet defined [16,19-24]. The use of triamcinolone injections in the esophagus has raised several concerns about the safety and effectiveness [18]. Moreover, systemic therapy with oral steroids is well known to have several side effects [25].

The question arises whether the use of topical steroids is effective in stricture prevention after ER of esophageal tumors, due to its effect in the suppression of the inflammatory process after ER [12]. Topical steroids have shown to be beneficial for eosinophilic esophagitis treatment and have resulted in stricture reduction after EMR of short Barrett's segment [12,26].

The aim of this study was to investigate the effectiveness of oral treatment with topical budesonide for the prevention of strictures after ER of EC (ESCC or esophageal adenocarcinoma $[E A C])$. We hypothesized a lower esophageal stricture rate when using topical budesonide compared to the stricture rate in patients who did not receive a preventive treatment after $E R$, reported in the literature. The secondary aim of this study was to investigate whether the use of topical budesonide after ER affects esophageal stricture treatment. We hypothesized that less dilations were required in patients treated with topical budesonide who developed a stricture after ER compared to patients who did not receive preventive treatment after $E R$, reported in the literature.

\section{Patient and methods Study design}

We performed a single-center, retrospective analysis of data that has been prospectively collected in our ongoing registry of ER of the esophagus at the Department of Gastroenterology and Hepatology at the Erasmus MC, University Medical Center. Patients treated with ER (EMR or ESD) for superficial ESCC or EAC between March 2015 and April 2020 were retrospectively included in this study. All patients that were deemed to have a high chance of stricture development (i.e. patients with a mucosal defect size after ER $>50 \%$ of the esophageal circumference) were treated with topical budesonide after ER in order to prevent stricture development [15]. This was decided directly after ER by the treating endoscopist [A.K]. Patients were excluded if they had known intolerance to budesonide, candida esophagitis, or immunocompromised conditions. Additionally, patients were excluded from final analysis if they were treated with prior ESD, EMR, radiotherapy, radiofrequency ablation, or endoscopic dilation in the area where the current ER was performed. These prior treatments could be of influence on stricture development. This study was approved by the Medical Ethical Review Committee of the Erasmus University MC in Rotterdam, the Netherlands [MEC-2019-0819].

\section{Data collection}

Data on patient and tumor characteristics were collected from medical charts and endoscopy and pathology reports. An overview of collected characteristics can be found in Supplementary File 1. If a patient developed a second (metachronous) tumor, the first tumor was included for final analysis to avoid bias.

ER was performed with either EMR or ESD, which was up to the discretion of the endoscopist or dictated by the tumor type. ESCC was always removed by ESD. EAC was only removed by ESD when submucosal invasion was expected. EMR was performed using the multiband mucosectomy method [27]. ESD was carried out with a HybridKnife (ERBE Elektromedizin $\mathrm{GmbH}$, Tuebingen, Germany), lifting fluid contained saline with indigo carmine and epinephrine [28]. All EMRs and ESDs were performed by a single endoscopist [A.K.].

\section{Treatment with budesonide after endoscopic resection}

The standard dose for topical budesonide was $2.3 \mathrm{mg}$ once a day, $2.3 \mathrm{mg}$ twice a day or $1 \mathrm{mg}$ twice a day, for a duration of 6 weeks from the first day after ER. In general, if adjuvant therapy (e. g. surgery or chemo-radiotherapy) was needed, budesonide was still given for 6 weeks because strictures can develop shortly after ER. For every patient, this was discussed in a multidisciplinary team. Only if adjuvant therapy started within this 6week period, budesonide treatment discontinued earlier. The budesonide dose depended on the availability of topical budesonide in the pharmacy. In 2015, $2.3 \mathrm{mg}$ budesonide once a day was given, and $2.3 \mathrm{mg}$ budesonide twice a day was given beginning in 2016. In the last 6 months of inclusion, orodispersible topical budesonide tablets of $1 \mathrm{mg}$ became available for eosinophilic esophagitis (EoE) treatment wherefore this dose was chosen. Because no orodispersible budesonide tablets were available for most of the study, the dispersible tablet from a budesonide enema was used for oral intake. Patients were instructed to let the tablet dissolve on the tongue and swallow the dispersed budesonide. This is a common off-label use of budesonide in the treatment of EoE. Recently, a budesonide orodispersible tablet has become available for treatment of EoE [29,30]. 


\section{Endoscopic dilation}

Endoscopic dilation was only performed when the patient had dysphagia in combination with esophageal stenosis. Prophylactic dilation was not performed. The type and interval of endoscopic dilation was up to the discretion of the endoscopist. In general, two dilation techniques were used: bougie and balloon dilation [31]. Dilations were usually done with a weekly repetition if necessary.

\section{Study endpoints}

The primary endpoint was the esophageal stricture rate in patients who received topical budesonide therapy after ER of early EC. A stricture was defined as the inability to swallow solid food and/or the inability to pass a standard diagnostic endoscope (diameter: 9.9 mm, GIF-H190 and GIF-H180 J, Olympus) which resulted in the need for dilation. We only included strictures that developed before adjuvant treatment (e.g. radiotherapy, radiofrequency ablation, EMR, chemotherapy) started. Stricture development after adjuvant treatment could also be attributed to the adjuvant treatment. Therefore, the date of adjuvant therapy was considered as the last moment of follow-up in these patients and strictures after that date we not included. Stricture rates in our cohort were compared with stricture rates of comparable control groups in the literature. Secondary endpoints included identification of risk factors for stricture development, number of endoscopic dilations per patient, type of dilation (balloon/bougie), time to dilation after ER (days), number of patients with dysphagia, number of patients who experienced budesonide side effects, and the number of patients with adverse events (AEs) after dilation, including the type of AEs. To investigate whether topical budesonide affects esophageal stricture treatment, the number of endoscopic dilations performed in patients who developed a stricture in our cohort was compared with the number of endoscopic dilations performed in patients who developed a stricture in control groups from the literature.

\section{Statistical analyses}

Continuous variables were presented with mean (range) and median (interquartile range (IQR)) for normally distributed and skewed data, respectively. Patients who developed a stricture were compared with patients who did not develop a stricture after ER to determine potential risk factors for stricture development. These two groups were compared with univariable analysis, performed by the independent Student's $t$-test for normally distributed continuous data and the Chi-square test for categorical data.

For the comparison of stricture rates and the number of dilations of patients in our cohort with patients who did not receive a preventive treatment after ER of EC, control groups of several studies were used in which other methods were investigated to prevent stricture development. For the selection of these control groups, a systematic literature search was performed in Pubmed and Medline by two independent reviewers (S.V. and M.S). The search strategy and selection of relevant literature is outlined in Supplementary File 2. Stricture rates in the control groups were calculated for each study as the total number of patients who developed a stricture in the control group divided by the total number of patients in the control group. We calculated the standard error (SE) for each study using the following formula: $S E=\sqrt{(s \cdot(1-s) / n)}$; where, $s=$ stricture rate and $n=$ total number of patients in the control group. A fixed-effects meta-analysis was performed to estimate the stricture rate of the control studies. To evaluate the heterogeneity between the studies, the inconsistency index $\left(I^{2}\right)$ was calculated [32]. The pooled stricture rate of the control studies was compared with the stricture rate of our cohort using the Z-test. We used descriptive statistics to compare the number of dilations of our study with the number of dilations in control studies; meta-analysis was not performed since all studies reported different values of the number of dilations (e.g. median in combination with range, mean in combination with $95 \% \mathrm{Cl}$, and no standard deviations were reported). For all analyses, a two-sided $P<0.05$ was considered significant. Statistical analyses were performed with the statistical software IBM SPSS Statistics (version 25) and Review Manager Software (version 5.3) was used for meta-analysis.

\section{Results}

A total of 64 patients were treated with ER for early EC. After exclusion of 22 patients (mucosal defect $<50 \%$; $n=16$, ESD performed in cardia; $n=6$ ), 42 patients received budesonide therapy after ER. One patient developed ESCC two times, only the first tumor was included for analysis to prevent bias. Baseline characteristics of all included patients are presented in - Table 1. Most patients were male $(n=25 ; 59.5 \%)$ and the median age was 67.0 years (IQR: $60.8-72.3$ ).

\section{Tumor and treatment characteristics}

Tumor and treatment characteristics are presented in $\downarrow$ Table 2 for 42 cases. Most tumors were located in the mid esophagus (14/42; 33.3\%) and lower esophagus (15/42; 35.7\%). The remaining tumors $(13 / 42 ; 31.0 \%)$ were overlapping between two sub-locations or were located in the upper thoracic esophagus. The median circumferential range of the mucosal defect after ER was $80.0 \%$ (IQR: 75.0-100.0). A total of four patients had a mucosal defect less than $75 \%$ and the smallest reported circumferential range was $60 \%$. The median surface of the resected specimen was $10.3 \mathrm{~cm}^{2}$ (IQR: 6.8-16.7). In total, 25/42 $(59.5 \%)$ tumors were ESCC and $17 / 42$ (40.5\%) tumors were EAC. There were four patients within the ESCC group with dysplasia; three with high-grade dysplasia and one with low-grade dysplasia. In total, 20 of 42 tumors showed submucosal invasion. In 16 cases, the absolute invasion depth was reported with a median of $975.0 \mu \mathrm{m}$ (IQR: 562.5-1725.0).

ESD was performed in $37(88.1 \%)$ cases and EMR was performed in five $(11.9 \%)$ cases. The dose of oral budesonide was $2.3 \mathrm{mg}$ twice a day (31/42 [73.8\%]), $2.3 \mathrm{mg}$ budesonide once a day (6/42 [14.3\%]) or $1 \mathrm{mg}$ budesonide twice a day $(5 / 42$ [11.9\%]). One patient discontinued budesonide treatment before the 6-week period was completed (reason unknown). This patient used budesonide for 2 weeks and developed a stricture 
- Table 1 Baseline characteristics of 42 patients and univariable analysis of the stricture group ( $n=18$ ) versus the non-stricture group ( $n=24)$.

\begin{tabular}{|c|c|c|c|c|}
\hline Characteristics & Total $(n=42)$ & Stricture $(n=18)$ & No stricture $(n=24)$ & $P$ value \\
\hline \multicolumn{4}{|l|}{ Sex, n (\%) } & \multirow[t]{3}{*}{0.86} \\
\hline - Female & $17(40.5 \%)$ & $7(41.2 \%)$ & $10(58.8 \%)$ & \\
\hline - Male & $25(59.5 \%)$ & $11(44.0 \%)$ & $14(56.0 \%)$ & \\
\hline Median age, years (IQR) & $67.0(60.8-72.3)$ & $66.0(60.8-72.5)$ & $67.0(60.5-72.3)$ & 0.96 \\
\hline \multicolumn{4}{|l|}{ ASA classification, n (\%) } & \multirow[t]{4}{*}{0.64} \\
\hline .1 & $4(9.5 \%)$ & $1(25.0 \%)$ & $3(75.0 \%)$ & \\
\hline . II & $25(59.5 \%)$ & $12(48.0 \%)$ & $13(52.0 \%)$ & \\
\hline$\cdot$. III & $13(31.0 \%)$ & $5(38.5 \%)$ & $8(61.5 \%)$ & \\
\hline \multicolumn{4}{|l|}{ Smoking status, n (\%) } & \multirow[t]{5}{*}{0.09} \\
\hline - Current & $12(31.6 \%)$ & $3(25.0 \%)$ & $9(75.0 \%)$ & \\
\hline - Former & $18(47.4 \%)$ & $8(44.4 \%)$ & $10(55.6 \%)$ & \\
\hline - Never & $8(21.1 \%)$ & $6(75.0 \%)$ & $2(25.0 \%)$ & \\
\hline - Missing & 4 & 1 & 3 & \\
\hline Median pack years (IQR) & $42.5(24.3-48.8)$ & $45.0(25.5-49.5)$ & $40.0(19.0-47.5)$ & \multirow[t]{2}{*}{0.64} \\
\hline - Missing & 22 & 7 & 15 & \\
\hline \multicolumn{4}{|l|}{ Alcohol consumption, n (\%) } & \multirow[t]{5}{*}{0.25} \\
\hline - Current & $25(64.1 \%)$ & $9(36.0 \%)$ & $16(64.0 \%)$ & \\
\hline - Former & $7(17.9 \%)$ & $3(42.9 \%)$ & $4(57.1 \%)$ & \\
\hline - Never & $7(17.9 \%)$ & $5(71.4 \%)$ & $2(28.6 \%)$ & \\
\hline - Missing & 3 & 1 & 2 & \\
\hline Median units alcohol/week (IQR) & $10.5(4.5-21.0)$ & $17.0(5.8-21.0)$ & $7(3.3-31.5)$ & \multirow[t]{2}{*}{0.57} \\
\hline - Missing & 12 & 6 & 6 & \\
\hline
\end{tabular}

- Table 2 Univariable analyses of tumor characteristics (42 tumors) and treatment characteristics between the stricture group ( $\mathrm{n}=18)$ and nonstricture group $(n=24)$.

\begin{tabular}{|c|c|c|c|c|}
\hline Characteristics & Total $(n=42)$ & Stricture $(n=18)$ & No stricture $(n=24)$ & $P$ value \\
\hline \multicolumn{4}{|l|}{ Tumor location, n (\%) } & \multirow{5}{*}{0.50} \\
\hline - Upper thoracic esophagus & 4 & $1(25.0 \%)$ & $3(75.0 \%)$ & \\
\hline - Mid thoracic esophagus & 14 & $7(50.0 \%)$ & $7(50.0 \%)$ & \\
\hline - Lower thoracic esophagus & 15 & $5(33.3 \%)$ & $10(66.7 \%)$ & \\
\hline - Overlapping & 9 & $5(55.6 \%)$ & $4(44.4 \%)$ & \\
\hline Median circumferential range of the mucosal defect after ER (\%) (IQR) & $80(75-100)$ & $100(75-100)$ & $75(75-88)$ & 0.01 \\
\hline \multicolumn{4}{|l|}{ Circumferential range of the mucosal defect after ER, $n(\%)$} & \multirow[t]{3}{*}{0.73} \\
\hline - $50-74 \%$ & 3 & $1(33.3 \%)$ & $2(66.6 \%)$ & \\
\hline - $75-100 \%$ & 39 & $17(43.6 \%)$ & $22(56.4 \%)$ & \\
\hline
\end{tabular}


- Table 2 (Continuation)

\section{Characteristics}

Total $(n=42)$

Stricture $(n=18)$

No stricture $(n=24)$

$P$ value

Morphology (Paris classification), n (\%)

- Protruded lesions

- Overlapping protruded/flat elevated lesions

- Flat elevated lesions

- Overlapping flat elevated/flat lesions

- Flat lesions

\begin{tabular}{|l|l|l|}
\hline 3 & $2(66.7 \%)$ & $1(33.3 \%)$ \\
\hline 8 & $4(50.0 \%)$ & $4(50.0 \%)$ \\
\hline 12 & $2(22.2 \%)$ & $10(83.3 \%)$ \\
\hline 12 & $7(58.3 \%)$ & $5(41.7 \%)$ \\
\hline 7 & $4(57.1 \%)$ & $3(42.9 \%)$ \\
\hline
\end{tabular}

Histology tumor, n (\%)

- SCC $^{1}$

- Adenocarcinoma

25

$12(48.0 \%)$

$13(52.0 \%)$

Differentiation grade, n (\%)

$6(35.3 \%)$

$11(64.7 \%)$

- Well/moderate [G1/G2]

- Poor [G3]

- Missing 2,3

25

17

$6(35.3 \%)$

Invasion depth, n (\%)

\begin{tabular}{|l|l|l|}
\hline 27 & $13(48.1 \%)$ & $14(51.9 \%)$ \\
\hline 10 & $3(30.0 \%)$ & $7(70.0 \%)$ \\
\hline 5 & 2 & 3 \\
\hline
\end{tabular}

- M2

- M3

- SM1

- SM2

- SM3

- SMx

- Missing 2,3

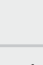

\begin{tabular}{|l|l}
\hline $0(0.0 \%)$ \\
\hline
\end{tabular}

$1(100.0 \%)$

16

$7(43.8 \%)$

$9(56.2 \%)$

\begin{tabular}{l|l}
1 & $1(100.0 \%)$
\end{tabular}

$0(0.0 \%)$

8

$4(50.0 \%)$

$4(50.0 \%)$

9

$2(22.2 \%)$

$7(77.8 \%)$

2

$2(100.0 \%)$

$0(0.0 \%)$

Median surface resection specimen, $\mathrm{cm}^{2}(\mathrm{IQR})$

- Missing

Median length of the resected specimen, $\mathrm{cm}(\mathrm{IQR})$

LVI present, $\mathrm{n}(\%)$

- Yes

- Missing ${ }^{3}$ 2

5

2

3

\begin{tabular}{|l|l|l|}
\hline $10.3(6.8-16.7)$ & $11.1(8.7-15.1)$ & $10.3(5.7-18.1)$
\end{tabular}

\begin{tabular}{l|l|l}
6 & 1 & 5
\end{tabular}

\section{5}

$4.5(3.5-5.4)$

$4.5(3.8-5.3)$

$4.4(3.3-5.7)$

0.95

0.70

Vertical resection margin, n (\%)

\begin{tabular}{|l|c|c|}
\hline - Positive [R1] & 10 & $4(40.0 \%)$ \\
\hline - Negative [R0] & 31 & $14(45.2 \%)$ \\
\hline - Missing & $17(54.8 \%)$ \\
\hline
\end{tabular}

Endoscopic resection method, n (\%)

$$
\text { - ESD }
$$

- EMR

37

$17(45.9 \%)$

$20(54.1 \%)$

\begin{tabular}{|l|l}
\hline 5 & $1(20.0 \%)$
\end{tabular}

$4(80.0 \%)$

Dose of budesonide, $\mathrm{n}(\%)$

- $2.3 \mathrm{mg} 2 \mathrm{dd}$ budesonide

- $2.3 \mathrm{mg} 1 \mathrm{dd}$ budesonide

- $1.0 \mathrm{mg} 2 \mathrm{dd}$ budesonide

\begin{tabular}{|l|l|l|}
\hline 31 & $13(41.9 \%)$ & $18(58.1 \%)$ \\
\hline 6 & $3(50.0 \%)$ & $3(50.0 \%)$ \\
\hline 5 & $2(40.0 \%)$ & $3(60.0 \%)$ \\
\hline
\end{tabular}

ER, endoscopic resection; IQR, interquartile range; EC, esophageal carcinoma; SCC, squamous cell carcinoma; LVI, lymphovascular invasion; EMR, endoscopic mucosal resection; ESD, endoscopic submucosal dissection.

${ }^{1}$ Including four patients with high-grade dysplasia $(n=3)$ or low-grade dysplasia $(n=1)$.

${ }^{2}$ Not reported for patients with high grade dysplasia $(n=3)$ or low-grade dysplasia $(n=1)$.

${ }^{3}$ Resection specimen was lost for pathology review in one patient. 


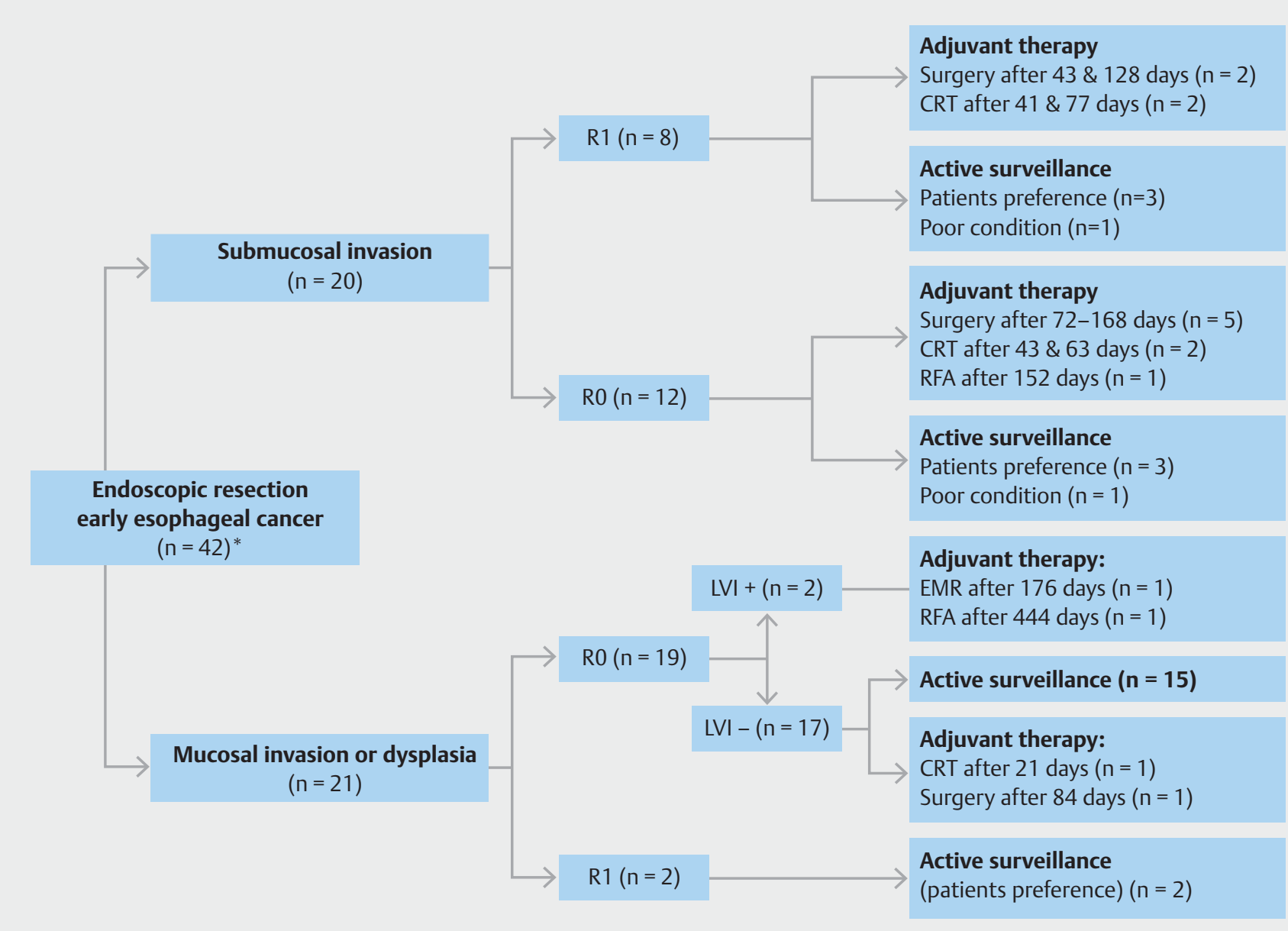

- Fig. 1 Adjuvant treatment after endoscopic resection of early esophageal cancer.

CRT, chemoradiotherapy; EMR, endoscopic mucosal resection; LVI, lymphovascular invasion; RFA, radiofrequency ablation.

*Resection specimen lost for pathology review in one patient; active surveillance was performed.

2 weeks thereafter. Overall, no side effects associated with topical budesonide were reported.

\section{Adjuvant treatment}

In total, 16 patients received adjuvant treatment after ER ( Fig. 1). The median time between ER and adjuvant treatment was 81 days (IQR: 48-147). In one patient, adjuvant treatment started 21 days after ER, before budesonide therapy was completed. In that patient, the ER specimen showed mucosal ESCC with LVI. This patient had two synchronous head and neck tumors, therefore, adjuvant radiotherapy was started for both esophageal and head and neck tumors.

\section{Stricture rate}

Eighteen of 42 patients (44.9\%) developed a stricture during follow-up. Patients with a mucosal defect of $\geq 75 \%$ of the esophageal circumference had a stricture rate of $43.6 \%$ (17/ 39) ( $\triangleright$ Table 2$)$. In this group $(n=39)$ there were 16 cases of EAC and 23 cases of ESCC. The stricture rate for patients with EAC in this group was $37.5 \%(6 / 16)$ and for patients with ESCC $47.8 \%(11 / 23)$.
A total of nine studies were selected from the literature (Supplementary File 2) [19, 21-24,33-36]. Patients in control groups from these studies all had ESCC and a mucosal defect after ER with a circumference $\geq 75 \%$. All studies were performed in Asia ( 5 in Japan, 3 in China, and 1 in Korea). In total, 104 of 147 patients who did not receive preventive treatment after ER developed a stricture. The stricture rate for these control groups ranged from $50.0 \%$ to $91.7 \%$. Meta-analysis with fixed-effect model was used to calculate the pooled stricture rate since $P^{2}$ was $44 \%$ (low heterogeneity). The pooled stricture rate was $75.3 \%(95 \% \mathrm{Cl} 68.6 \%-81.9 \%$ ) ( $\mathbf{F i g . 2}$ ). The stricture rate in our cohort of patients with ESCC and a mucosal defect after ER with a circumference $\geq 75 \%$ was $47.8 \%$ and was significantly lower compared with the control groups $(47.8 \%$ vs. $75.3 \%, P=0.007)$.

\section{Potential risk factors for stricture development}

Patients who developed a stricture were compared with patients who did not develop a stricture. The stricture group consisted of 18 of 42 patients (44.9\%) whereas the non-stricture group consisted of 24 of 42 patients (57.1\%). There was no sig- 


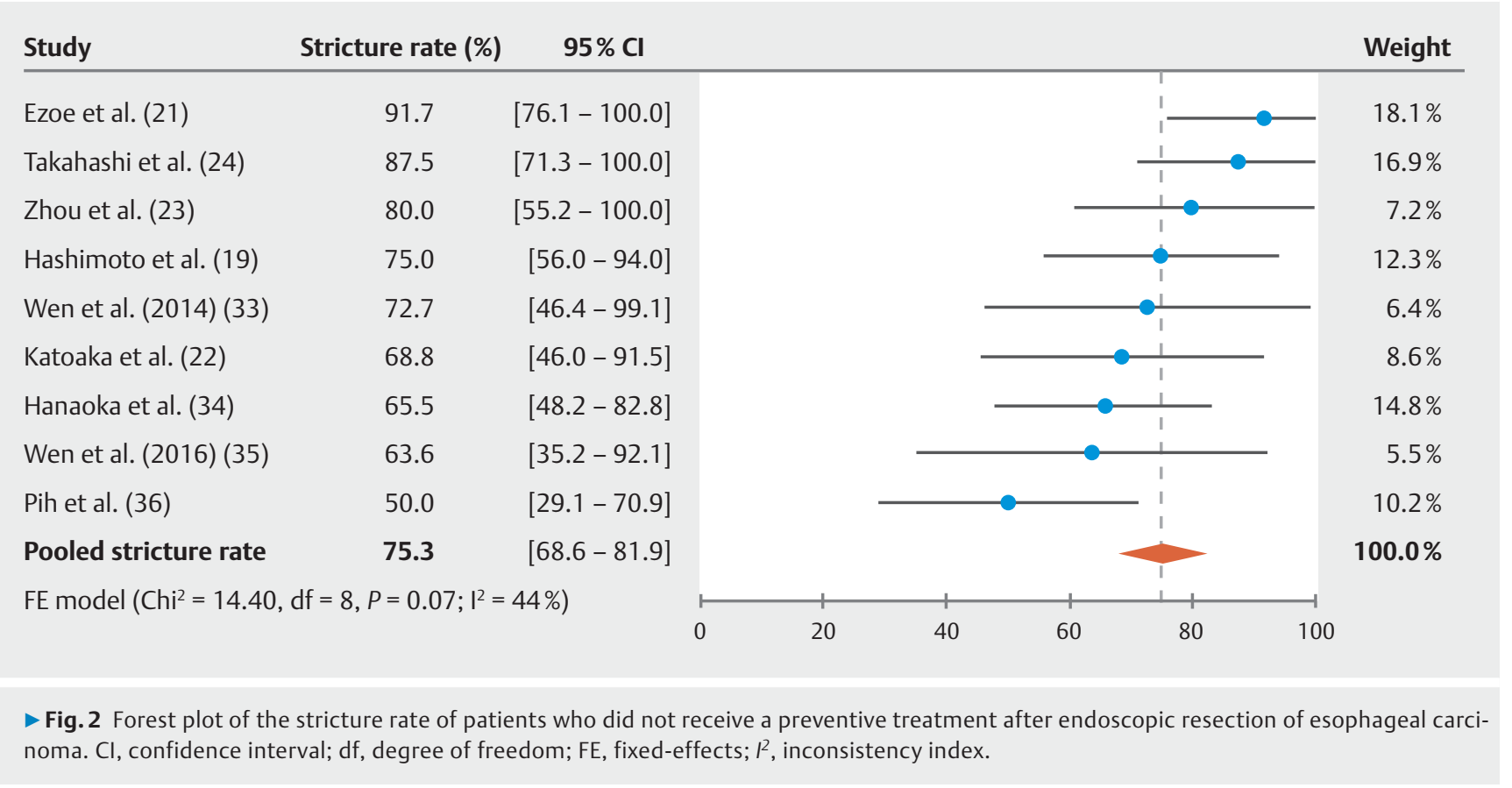

nificant difference in sex, age, smoking status, alcohol consumption, or American Society of Anesthesiologists classification between the two groups ( $\triangleright$ Table 1 ). The median circumferential range of the mucosal defect in the stricture group was $100.0 \%$ compared with $75.0 \%$ in the non-stricture group $(P=0.02)$ ( Table 2).

\section{Stricture development and dilations}

The median follow-up time for patients who developed a stricture $(n=18)$ was 53.4 weeks (IQR: $17.7-79.5)$. Dysphagia was reported in 17 of 18 patients (94.4\%). In total, 147 dilations were performed. The median number of endoscopic dilations per patient was 6.0 (IQR: 4.0-14.0). The median number of dilations in patients with ESCC and a mucosal defect after ER with a circumference $\geq 75 \%$ was also 6.0 (IQR 2-16). In case of a stricture, bougie dilation $(116 / 147 ; 78.9 \%)$ was more often used compared with $\operatorname{EBD}(31 / 147 ; 21.1 \%)$. The median time to dilation after ER was 29.0 (IQR: 20.0-44.5) days. Two patients developed an AE after dilation. One patient had a poor healing ulcer after dilation, which was successfully treated with pantoprazole. Another patient developed a perforation, which was treated with stent placement. The patient was hospitalized for 2 days for observation without further events. The stent was removed after 4 weeks and the perforation had healed.

The median number of dilations in patients who developed a stricture in control groups was reported in three studies; 8.1 (range 1-18), 4.5 (range 2-35) and 2 (range 0-15)[21, 22, 34]. Other studies reported the mean number of dilations; 12.5 (95\% Cl 7.1-17.9) in Takahashi et al., 6.6 (range 0-20) in Hashimoto et al, 3.9 (range $0-17$ ) in Wen et al. (2014) and 13.5 (range $0-28$ ) in Zhou et al $[19,23,24,33]$. No standard deviations were reported.

\section{Discussion}

ER of EC is an excellent minimally invasive treatment method to cure patients from early EC. A major disadvantage, however, is development of esophageal strictures after the procedure [10]. Most strictures have been observed when the mucosal defect after the procedure extends beyond $75 \%$ of the esophageal circumference $[10,13,14]$. We performed a retrospective analysis of a prospectively collected cohort of patients who received topical budesonide after ER of early EC to investigate whether use of topical budesonide prevents esophageal strictures after ER.

We found an overall stricture rate of $44.9 \%$ in patients who received 6-week treatment with topical budesonide after ER of early EC (both ESCC and EAC), compared with a pooled stricture rate of $75.3 \%$ when no preventive measures are taken as reported in the literature [19,21-24,33-36]. No side effects of budesonide were reported. All patients had an esophageal mucosal defect after ER with a circumference $\geq 60 \%$. The median circumference of the mucosal defect was higher in patients who developed a stricture compared to patients who did not develop a stricture $(100.0 \%$ versus $75.0 \%$; $P=0.02)$. All patients who developed a stricture were treated with endoscopic dilations, with a median time to dilation of 29.0 days (IQR 20.044.5 ) and the median number of dilations was 6.0 (IQR 4.014.0). There was only one perforation after dilation, which was successfully treated with stent placement.

The stricture rate in patients in our study with ESCC and a mucosal defect $\geq 75 \%$ of the esophageal circumference was $47.8 \%$, which is lower than the pooled stricture rate in patients who did not receive preventive treatment after ER (75.3\%; $P=$ 0.007). Topical budesonide therapy seems to be effective for stricture prevention after ER of early EC. The median number of dilations performed in patients who developed a stricture in our cohort (6.0) is in line with the median number of dilations, 
ranging from 2.0 to 8.1 , performed in patients who did not receive preventive treatment after ER [21, 22, 34]. However, only three studies reported the median number of dilations without a standard deviation or IQR. We could therefore not compare our results with control groups from the literature using metaanalysis. As a consequence, we could not investigate whether use of topical budesonide after ER affects esophageal stricture treatment.

Several studies have investigated different methods of preventing esophageal strictures after $E R$, such as preventive EBD, oral prednisolone, triamcinolone injections, and treatment with viscous budesonide slurry $[12,19,21-24]$. Patients in these studies had an esophageal mucosal defect with a circumference $\geq 75 \%$, comparable to our study. Although most of these studies reported a lower stricture rate in the treatment group compared to the stricture rate in our cohort, several limitations of these preventive methods are reported and all studies had small sample sizes with only 13 to 29 patients included in the treatment group [12,19,21-24]. The stricture rate in patients with ESCC who were treated with preventive EBD after ER was $59 \%$ compared to $92 \%$ in the control group $(P=0.04)$ [21]. There was no significant difference in the number of dilations after stricture development in the treatment group compared to the control group (2.0 vs. $4.5 ; P=0.05$ ) [21]. Patients in the treatment group received preventive EBD every week until complete healing of the mucosal defect was observed, which could be associated with patient burden and additional costs [21]. Treatment with oral prednisolone in ESCC patients was reported in two studies and resulted in a significantly lower stricture rate of $17.7 \%$ to $23.1 \%$ compared with $68.8 \%$ to $80.0 \%$ in the control groups $[22,23]$. In both studies, the number of required dilations was significantly higher in the control group compared to patients receiving oral prednisolone [22,23]. A disadvantage of systematic therapy with oral steroids are several side effects that may occur, such as immune suppression, infections, optical damage, and psychiatric disturbance [25]. Use of triamcinolone injections after ER in patients with ESCC resulted in a significantly lower stricture rate of $19.0 \%$ to $62.5 \%$ compared with $75.0 \%$ to $87.5 \%$ in control groups [19,24]. In both studies, fewer dilations were required in the treatment groups. A limitation of this invasive method are the extra required endoscopic procedures, causing additional costs and potential patient burden. Moreover, there is a risk of developing perforations after these injections [19]. Bahin et al. reported the effect of an oral treatment with viscous budesonide slurry (a mix of budesonide with sucralose) in patients with an EAC and a significant stricture reduction after EMR was observed compared to a control group ( $13.8 \%$ vs $37.3 \%, P<0.01)$ [12]. This treatment was only given to patients with an EAC, and patients with a Barrett segment larger than C3M5 were excluded [12].

This is the first study to investigate the effect of topical budesonide on stricture prevention after ER of early esophageal neoplasia. Our study suggests that use of topical budesonide is safe and effective for prevention of stricture after ER. Topical budesonide is a noninvasive treatment, which is a major strength of this study, and no side effects were reported. How- ever, our results have to be interpreted with caution due to several limitations. The first limitation is the retrospective design of our study, performed in a single center. We had missing data, which may have influenced our results and could have resulted in information bias. The second limitation is the small sample size of 42 patients, of whom 18 patients developed a stricture. Therefore, we could not perform multivariable risk factor analysis to adjust for confounders. The third limitation is the non-randomized study design without the availability of a control group. Because there was no control group, it is impossible to know whether use of topical budesonide was the main reason for the lower stricture rate. Another limitation is the potential for selection bias. The endoscopist decided whether patients received budesonide after ER, based on the estimated risk of developing strictures. Further, although patients all reported taking the medication correctly during follow-up, we did not have a formal procedure in place to confirm that. Because no topical budesonide tablets were available during the largest part of the study period, we prescribed the dispersible budesonide tablets from a budesonide enema. This off-label use could result in incorrect use of budesonide. Moreover, different doses of budesonide were used during the study period. It seems likely that an orodispersible tablet designed for this indication could yield an even higher effect in prevention of strictures. To address these limitations, a prospective, randomized controlled trial (RCT) is necessary to investigate the efficacy and tolerability of budesonide orodispersible tablets.

\section{Conclusion}

In conclusion, based on comparisons with historical published data, topical budesonide after ER for EC seems to be an effective method for preventing stricture development. The stricture rate was lower compared with the rates in patients who did not receive a preventive method after ER. However, a prospective RCT is required to investigate whether topical budesonide is safe and effective for prevention of strictures after ER in patients with early stage EC, and whether topical budesonide affects esophageal stricture treatment.

\section{Competing interests}

The authors declare that they have no conflict of interest.

References

[1] Bray F, Ferlay J, Soerjomataram I et al. Global cancer statistics 2018 GLOBOCAN estimates of incidence and mortality worldwide for 36 cancers in 185 countries. CA Cancer J Clin 2018; 68: 394-424

[2] Thun MJ, Linet MS, Cerhan JR. Cancer Epidemiology and Prevention. Oxford University Press; 2018

[3] van Putten M, de Vos-Geelen J, Nieuwenhuijzen GAP et al. Long-term survival improvement in oesophageal cancer in the Netherlands. Eur J Cancer 2018; 94: 138-147

[4] Shapiro J, van Lanschot JJB, Hulshof M et al. Neoadjuvant chemoradiotherapy plus surgery versus surgery alone for oesophageal or 
junctional cancer (CROSS): long-term results of a randomised controlled trial. Lancet Oncol 2015; 16: 1090-1098

[5] Moss A, Bourke M], Hourigan LF et al. Endoscopic resection for Barrett's high-grade dysplasia and early esophageal adenocarcinoma: an essential staging procedure with long-term therapeutic benefit. Am J Gastroenterol 2010; 105: 1276-1283

[6] Takahashi H, Arimura Y, Masao H et al. Endoscopic submucosal dissection is superior to conventional endoscopic resection as a curative treatment for early squamous cell carcinoma of the esophagus (with video). Gastrointest Endosc 2010; 72: 255-264, 264 e251-252

[7] Pimentel-Nunes P, Dinis-Ribeiro M, Ponchon T et al. Endoscopic submucosal dissection: European Society of Gastrointestinal Endoscopy (ESGE) Guideline. Endoscopy 2015; 47: 829-854

[8] Fujishiro M, Yahagi N, Kakushima $\mathrm{N}$ et al. En bloc resection of a large semicircular esophageal cancer by endoscopic submucosal dissection. Surg Laparosc Endosc Percutan Tech 2006; 16: 237-241

[9] Ono S, Fujishiro M, Niimi K et al. Long-term outcomes of endoscopic submucosal dissection for superficial esophageal squamous cell neoplasms. Gastrointest Endosc 2009; 70: 860-866

[10] Shi Q, Ju H, Yao LQ et al. Risk factors for postoperative stricture after endoscopic submucosal dissection for superficial esophageal carcinoma. Endoscopy 2014; 46: 640-644

[11] Tsujii Y, Nishida T, Nishiyama O et al. Clinical outcomes of endoscopic submucosal dissection for superficial esophageal neoplasms: a multicenter retrospective cohort study. Endoscopy 2015; 47: 775-783

[12] Bahin FF, Jayanna M, Williams S] et al. Efficacy of viscous budesonide slurry for prevention of esophageal stricture formation after complete endoscopic mucosal resection of short-segment Barrett's neoplasia. Endoscopy 2016; 48: 71-74

[13] Katada C, Muto M, Manabe T et al. Esophageal stenosis after endoscopic mucosal resection of superficial esophageal lesions. Gastrointest Endosc 2003; 57: 165-169

[14] Ono S, Fujishiro M, Niimi K et al. Predictors of postoperative stricture after esophageal endoscopic submucosal dissection for superficial squamous cell neoplasms. Endoscopy 2009; 41: 661-665

[15] Lewis JJ, Rubenstein JH, Singal AG et al. Factors associated with esophageal stricture formation after endoscopic mucosal resection for neoplastic Barrett's esophagus. Gastrointest Endosc 2011; 74: 753-760

[16] lizuka T, Kikuchi D, Yamada A et al. Polyglycolic acid sheet application to prevent esophageal stricture after endoscopic submucosal dissection for esophageal squamous cell carcinoma. Endoscopy 2015; 47: 341-344

[17] Takahashi H, Arimura Y, Okahara S et al. Risk of perforation during dilation for esophageal strictures after endoscopic resection in patients with early squamous cell carcinoma. Endoscopy 2011; 43: 184189

[18] Barret M, Beye B, Leblanc S et al. Systematic review: the prevention of oesophageal stricture after endoscopic resection. Aliment Pharmacol Ther 2015; 42: 20-39

[19] Hashimoto S, Kobayashi M, Takeuchi M et al. The efficacy of endoscopic triamcinolone injection for the prevention of esophageal stricture after endoscopic submucosal dissection. Gastrointest Endosc 2011; 74: 1389-1393
[20] Sakaguchi Y, Tsuji Y, Ono S et al. Polyglycolic acid sheets with fibrin glue can prevent esophageal stricture after endoscopic submucosal dissection. Endoscopy 2015; 47: 336-340

[21] Ezoe Y, Muto M, Horimatsu T et al. Efficacy of preventive endoscopic balloon dilation for esophageal stricture after endoscopic resection. J Clin Gastroenterol 2011; 45: 222-227

[22] Kataoka M, Anzai S, Shirasaki T et al. Efficacy of short period, low dose oral prednisolone for the prevention of stricture after circumferential endoscopic submucosal dissection (ESD) for esophageal cancer. Endosc Int Open 2015; 3: E113-117

[23] Zhou G, Yuan F, Cai J et al. Efficacy of prednisone for prevention of esophageal stricture after endoscopic submucosal dissection for superficial esophageal squamous cell carcinoma. Thorac Cancer 2017; 8: 489-494

[24] Takahashi H, Arimura Y, Okahara S et al. A randomized controlled tria of endoscopic steroid injection for prophylaxis of esophageal stenoses after extensive endoscopic submucosal dissection. BMC Gastroenterol 2015; 15: 1

[25] Melillo N, Corrado A, Quarta L et al. Corticosteroids, a review. Panminerva Med 2007; 49: 29-33

[26] Lucendo AJ, Miehlke S, Schlag C et al. Efficacy of Budesonide Orodispersible Tablets as Induction Therapy for Eosinophilic Esophagitis in a Randomized Placebo-Controlled Trial. Gastroenterology 2019; 157: 74-86 e15

[27] Pouw RE, van Vilsteren FG, Peters FP et al. Randomized trial on endoscopic resection-cap versus multiband mucosectomy for piecemeal endoscopic resection of early Barrett's neoplasia. Gastrointest Endosc 2011; 74: 35-43

[28] Park CH, Shin S, Lee SK et al. Assessing the stability and safety of procedure during endoscopic submucosal dissection according to sedation methods: a randomized trial. PLoS One 2015; 10: e0120529

[29] Miehlke S, Hruz P, Vieth M et al. A randomised, double-blind trial comparing budesonide formulations and dosages for short-term treatment of eosinophilic oesophagitis. Gut 2016; 65: 390-399

[30] Straumann A, Conus S, Degen L et al. Budesonide is effective in adolescent and adult patients with active eosinophilic esophagitis. Gastroenterology 2010; 139: 1526-1537, 1537 e1521

[31] Pasha SF, Acosta RD. ASGE Standards of Practice Committee. et al. The role of endoscopy in the evaluation and management of dysphagia. Gastrointest Endosc 2014; 79: 191-201

[32] Higgins JP, Thompson SG, Deeks J] et al. Measuring inconsistency in meta-analyses. BMJ 2003; 327: 557-560

[33] Wen J, Lu Z, Yang Y et al. Preventing stricture formation by covered esophageal stent placement after endoscopic submucosal dissection for early esophageal cancer. Dig Dis Sci 2014; 59: 658-663

[34] Hanaoka N, Ishihara R, Takeuchi Y et al. Intralesional steroid injection to prevent stricture after endoscopic submucosal dissection for esophageal cancer: a controlled prospective study. Endoscopy 2012; 44: 1007-1011

[35] Wen J, Lu Z, Linghu E et al. Prevention of esophageal strictures after endoscopic submucosal dissection with the injection of botulinum toxin type A. Gastrointest Endosc 2016; 84: 606-613

[36] Pih GY, Kim DH, Gong EJ et al. Preventing esophageal strictures with steroids after endoscopic submucosal dissection in superficial esophageal neoplasm. J Dig Dis 2019; 20: 609-616 